\title{
Tooth discoloration induced by a novel mineral trioxide aggregate-based root canal sealer
}

\author{
Dae-Sung Lee ${ }^{1}$, Myung-Jin Lim ${ }^{1}$, Yoorina Choi ${ }^{2}$, Vinicius Rosa ${ }^{3}$, Chan-Ui Hong ${ }^{4}$, \\ Kyung-San Min ${ }^{1,5}$
}

Correspondence: Dr. Kyung-San Min

Email: endomin@gmail.com

\begin{abstract}
'Department of Conservative Dentistry, School of Dentistry, Chonbuk National University, Jeonju, Korea, ${ }^{2}$ Department of Conservative Dentistry, Wonkwang University Dental Hospital, Iksan, Korea, ${ }^{3}$ Discipline of Oral Sciences, Faculty of Dentistry, National University of Singapore, Singapore, ${ }^{4}$ Department of Conservative Dentistry, School of Dentistry, Dankook University, Cheonan, Korea, ${ }^{5}$ Biomedical Research Institute of Chonbuk National University Hospital, Jeonju, Korea
\end{abstract}

\section{ABSTRACT}

Objectives: The aim of this study was to evaluate tooth discoloration caused by contact with a novel injectable mineral trioxide aggregate (MTA)-based root canal sealer (Endoseal; Maruchi, Wonju, Korea) compared with a widely used resin-based root canal sealer (AHplus; Dentsply De Trey, Konstanz, Germany) and conventional MTA (ProRoot; Dentsply, Tulsa, OK, USA). Materials and Methods: Forty standardized bovine tooth samples were instrumented and divided into three experimental groups and one control group ( $n=10$ /group). Each material was inserted into the cavity, and all specimens were sealed with a self-adhesive resin. Based on CIE Lab system, brightness change $(\Delta L)$ and total color change $(\Delta E)$ of each specimen between baseline and 1, 2, 4, and 8 weeks were obtained. Results: At all time points, Endoseal showed no significant difference in $\Delta L$ and $\Delta E$ compared to AHplus and control group $(P>0.05)$, whereas the ProRoot group showed significantly higher $\Delta L$ and $\Delta E$ values than the Endoseal group at 2, 4, and 8 weeks $(P<0.05)$. Therefore, Endoseal showed less discoloration than conventional MTA and a similar color change to AHplus. Conclusions: Within the limitations of this study, our data indicate that the MTA-based sealer produces a similar amount of tooth discoloration as AHplus which is considered to be acceptable.

Key words: Mineral trioxide aggregate, root canal sealer, spectrophotometry, tooth discoloration

\section{INTRODUCTION}

Root canal sealers are generally used in combination with Gutta-percha to seal the root canal system. These materials are categorized according to their main chemical composition such as zinc oxide eugenol, calcium hydroxide, epoxy resin, or glass ionomer. ${ }^{[1]}$ As the root canal sealers are put into the canal during the filling procedure, there is a possibility that some

\begin{tabular}{|l|l|}
\hline \multicolumn{2}{|c|}{ Access this article online } \\
\hline Quick Response Code: \\
\hline
\end{tabular}

portion of the filler remains smeared in the coronal access cavity despite cleaning with alcohol pellets or careful preparation of the cavity. Therefore, it is important to predict how much discoloration would occur if root canal sealer is left in the access cavity. Furthermore, the color of the root canal sealer itself may produce tooth discoloration. This discoloration

This is an open access article distributed under the terms of the Creative Commons Attribution-NonCommercial-ShareAlike 3.0 License, which allows others to remix, tweak, and build upon the work non-commercially, as long as the author is credited and the new creations are licensed under the identical terms.

For reprints contact: reprints@medknow.com

How to cite this article: Lee DS, Lim MJ, Choi Y, Rosa V, Hong CU, Min KS. Tooth discoloration induced by a novel mineral trioxide aggregatebased root canal sealer. Eur J Dent 2016;10:403-7.

DOI: $10.4103 / 1305-7456.184165$ 
may be seen in the cervical third of the crown where the overlying enamel, which is a translucent and colorless structure, is thinner. ${ }^{[2]}$ Therefore, improper coronal extension of the root canal filling above the gingival margin should be avoided.

Mineral trioxide aggregate (MTA) is a useful material that was first introduced for the purpose of root-end filling. ${ }^{[3]}$ Numerous in vitro and in vivo studies have confirmed its superior properties such as biocompatibility, bioactivity, and sealability. ${ }^{[4-6]}$ Therefore, MTA has been advocated for use in various clinical procedures including pulp capping, pulpotomy, apexification, and perforation repair. Furthermore, there have been attempts to use MTA as a root canal filling material and some MTA-based products have been introduced into the endodontic market. Regardless of the composition, most of the currently used sealer systems consist of a powder/liquid or base/catalyst and these two components must be mixed at chairside and then applied to the root canal coated with Gutta-percha. During this procedure, the sealer may contaminate the pulp chamber and any remaining sealer may induce tooth discoloration.

Recently, a novel root canal sealer based on MTA (EndoSeal; Maruchi, Wonju, Korea) has been developed in an attempt to introduce the useful features of MTA into the root canal sealer. Endoseal is a premixed and injectable endodontic sealer that uses moisture in the air to initiate the setting reaction [Figure 1]. Consequently, it sets slowly by itself without any mixing procedure. A recent study indicates that Endoseal has comparable physical properties to MTA and superior biocompatibility compared to AHplus. ${ }^{[7]}$ However, many studies show that MTA, which is mainly composed of calcium silicate and bismuth oxide, has discoloration potential. ${ }^{[8-10]}$ Naik and Hegde reported that when MTA was used for pulpotomy in

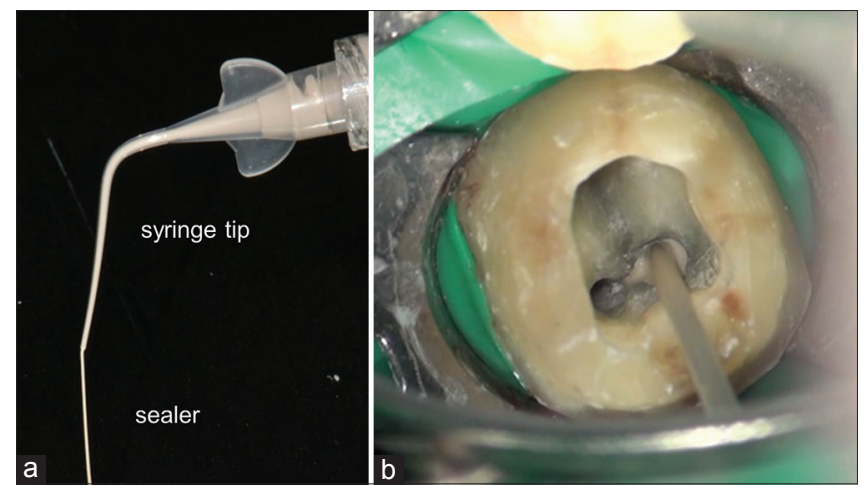

Figure 1: Endoseal (a) and its clinical application (b) primary molars, discoloration occurred in $60 \%$ of all cases. ${ }^{[11]}$ Belobrov and Parashos also presented a case report of a complicated crown fracture treated by partial pulpotomy with white MTA that resulted in tooth discoloration. ${ }^{[12]}$ Therefore, when dealing with any MTA-based sealer the potential of discoloration of the tooth cannot be excluded. However, limited information is available regarding the effect of this new root canal sealer on tooth discoloration. The purpose of this in vitro study was to evaluate the tooth discoloration effect of Endoseal in comparison with a commonly used root canal sealer (AHplus) and conventional MTA (ProRoot). The null hypothesis was as follows: There is no difference between the tested materials regarding tooth discoloration.

\section{MATERIALS AND METHODS}

\section{Sample preparation}

A total of 40 intact bovine incisors were used. Exclusion criteria were the presence of caries, coronal staining, observable structural defects, and narrow crown width and height (each should be longer than $10 \mathrm{~mm}$ ). The samples were prepared as shown in Figure 2a with reference to the model introduced in previous studies. ${ }^{[8,13]}$ In brief, bovine teeth were disinfected in $1 \%$ chloramine-T solution (Sigma-Aldrich, St. Louis, MO, USA) and stored in normal saline at room temperature for 30 days. After resection of the roots with a diamond-coated disc, an ultrasonic scaler was used to remove the extrinsic stains and calculus on the coronal labial surface.

Using a microtome (ISOMET; Buehler, Lake Bluff, IL, USA), a cuboid enamel-dentin block $(10 \mathrm{~mm} \times 10 \mathrm{~mm} \times 3.5 \mathrm{~mm})$ was obtained from the middle third of each crown. The labial enamel surface was finished and polished with successive use of 220,600, 1200, and 2000 grit abrasive papers (CC261; DEERFOS, Seoul, Korea). A box-form cavity

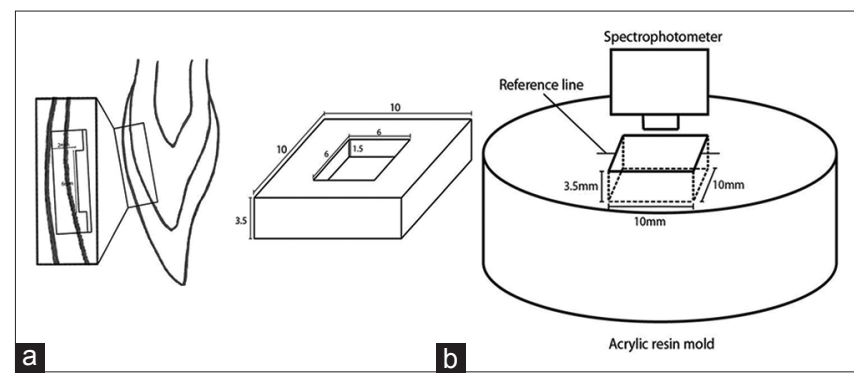

Figure 2: (a) Standardized cuboid enamel-dentin block prepared by removing the middle third of a bovine incisor. (b) Standardized acrylic resin mold used to repeatedly measure the same position in each sample 
$(6 \mathrm{~mm} \times 6 \mathrm{~mm} \times 1.5 \mathrm{~mm})$ was prepared with a diamond bur in the middle of each specimen, leaving approximately $2 \mathrm{~mm}$ thickness of the labial tooth structure ( $1 \mathrm{~mm}$ each of enamel and dentin). Solutions of $2 \%$ sodium hypochlorite $(\mathrm{NaOCl})$ and $17 \%$ ethylene diamine tetraacetic acid (EDTA; PrevestDentpro, Jammu, India) were applied to the specimens for $30 \mathrm{~min}$ and $2 \mathrm{~min}$, respectively. A final rinse was performed with $1 \% \mathrm{NaOCl}$ and saline. All specimens were stored at room temperature and $100 \%$ relative humidity.

\section{Experimental and control groups}

The specimens were randomly assigned to three experimental groups and one negative control group $(n=10)$. Each material was mixed according to the manufacturers' instructions and placed into the tooth cavity of the relevant group; nothing was placed in the cavity for the control group. A resin material (RelyX Unicem; 3M ESPE, Seefeld, Germany) was used to seal all of the cavities. All specimens were stored at room temperature and $100 \%$ relative humidity.

\section{Tooth color measurement}

A standardized acrylic resin mold was constructed for measurement with a spectrophotometer (Color i5; GretagMacbeth, Martinsried, Germany) [Figure 2b]. This mold allowed each specimen to be measured in the same position each time. The sample was positioned in the mold and the spectrophotometer was adjusted to the reference line. The tooth color measurement was taken at baseline $\left(\mathrm{W}_{0}\right.$; immediately after tooth preparation and placement of materials) and at $1\left(\mathrm{~W}_{1}\right), 2\left(\mathrm{~W}_{2}\right), 4\left(\mathrm{~W}_{4}\right)$, and 8 weeks $\left(\mathrm{W}_{8}\right)$ with a spectrophotometer. All measurements were repeated three times and averaged.

The difference in brightness $(\Delta L)$ at each time point was calculated by subtracting the corresponding $L$ value from the baseline $L$ value. The color difference $(\Delta E)$ between the baseline and the $W_{1^{\prime}}, W_{2^{\prime}}, W_{4^{\prime}}$ and $W_{8}$ measurements was calculated using the following formula:

$\Delta E=\left(\left[L^{*} 2-L^{*} 1\right]^{2}+\left[a^{*} 2-a^{*} 1\right]^{2}+\left[b^{\star} 2-b^{*} 1\right]^{2}\right)^{1 / 2}$

where $L^{*}$ represents the degree of lightness and ranges from 0 (black) to 100 (white), a* represents degree of greenness (negative $a^{*}$ ) or redness (positive $a^{*}$ ), and $b^{*}$ represents degree of blueness (negative $b^{*}$ ) or yellowness (positive $b^{*}$ ). ${ }^{[14]}$

\section{Statistical analysis}

SPSS software (SPSS 12.0K for Windows; SPSS Inc., Chicago, IL, USA) was used to evaluate the data. One-way analysis of variance and Tukey's post hoc test were used to evaluate significant differences between the tested materials at each time point $(P=0.05)$.

\section{Stereomicroscopic examination}

A representative sample was randomly selected for each group and sectioned horizontally at $1 \mathrm{~mm}$ thickness with a low-speed microtome (ISOMET). The slice in the center of the sample was selected and the cross section was examined under a stereomicroscope (Leica MZ16FA; Leica, Wezler, Germany).

\section{RESULTS}

The tooth color measurement data are summarized in Figures 3 and 4. The Endoseal group showed a similar amount of brightness change $(\Delta L)$ and color change $(\Delta E)$ as the AHplus group. At 1 week, the ProRoot group showed significantly higher $\Delta L$ compared to the ES group $(P<0.05)$. However, the AHplus and control group showed no significant difference from the other groups $(P>0.05)$. At 2 weeks, the $\Delta L$ and $\Delta E$ values of the ProRoot group increased and as a result, the ProRoot group showed a significant difference from all the other groups $(P<0.05)$. At 4 weeks, the ProRoot group still showed a significantly higher $\Delta L$ and $\Delta E$ than all other groups $(P<0.05)$, whereas the Endoseal group showed no significant difference from the AHplus and control group $(P>0.05)$. At 8 weeks, the ProRoot group showed significantly higher $\Delta L$ and $\Delta E$ than the Endoseal and control group $(P<0.05)$. The Endoseal group was not significantly different from the AHplus and control group for both $\Delta L$ and $\Delta E(P>0.05)$. On stereomicroscopic examination, a dark discolored area was shown in dentin in contact with ProRoot, but not in any other group [Figure 5].

Overall, whereas ProRoot tended to show the greatest brightness or total color change, the change in the Endoseal group tended to remain relatively low, comparable to that in the AHplus and control groups. At all time points, Endoseal showed no significant difference from the control group in both brightness difference and total color difference. Endoseal also showed no significant difference from the AHplus group at all time points. Therefore, Endoseal shows a similarly small amount of tooth discoloration to AHplus, and was comparable to the control where no sealer was applied on the cavity.

\section{DISCUSSION}

Although MTA has favorable physical and biological properties, attempts to insert MTA as a root canal 


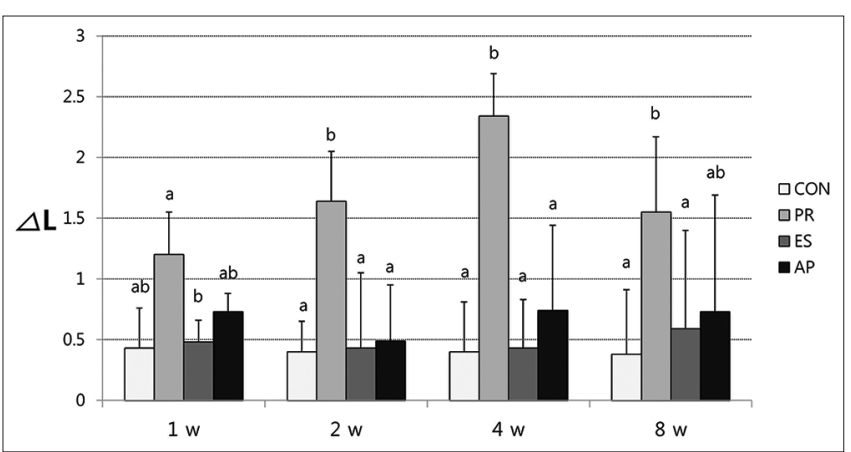

Figure 3: $\Delta L$ values (mean \pm standard deviation) for each group at five different time points. The same letters indicate no significant difference between the groups (Tukey test, $P=0.05$ ). CON: Control, PR: ProRoot, ES: Endoseal, AP: AHplus

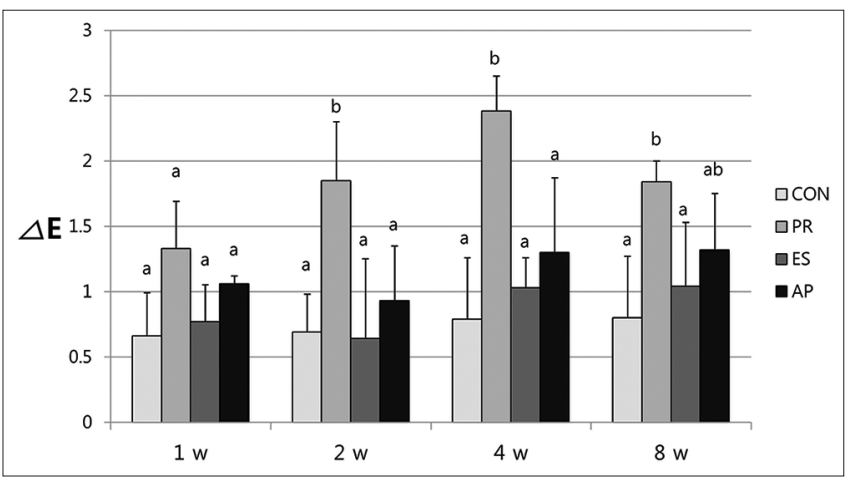

Figure 4: $\Delta E$ values (mean \pm standard deviation) for each group at five different time points. The same letters indicate no significant difference between groups (Tukey test, $P=0.05)$. CON: Control, PR: ProRoot, ES: Endoseal, AP: AHplus

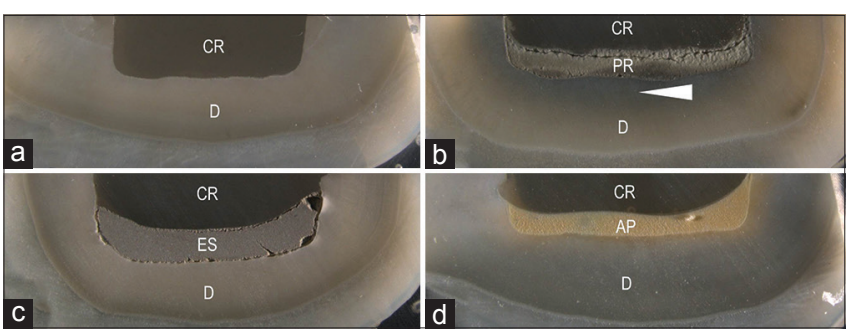

Figure 5: Stereomicroscopic appearance of a representative sample from each group after 8 weeks. (a) Control, (b) ProRoot, (c) Endoseal, and (d) AHplus. CR: Composite resin, D: Dentin, PR: ProRoot, ES: Endoseal, AP: AHplus. The white arrow indicates a discolored area induced by ProRoot

sealer have been hampered by its poor manipulability. Recently, a premixed, injectable endodontic sealer (Endoseal) was introduced to the endodontic field. This injectable MTA-based sealer is preserved in an airtight syringe and applied into the root canal by injection. Consequently, clinicians can easily apply the sealer directly into the root canal without contaminating the access cavity. Furthermore, it was recently demonstrated that Endoseal has favorable physical characteristics and biocompatibility. ${ }^{[7]}$ However, a controversy arose regarding tooth discoloration because the base material of Endoseal is MTA, which is known to induce discoloration.

In the present study, Endoseal showed significantly lower $\Delta L$ and $\Delta E$ values compared to ProRoot, a widely used conventional MTA $(P<0.05)$. Furthermore, Endoseal did not show any difference in $\Delta L$ and $\Delta E$ compared with AHPlus, and even with the control $(P>0.05)$. Consequently, our null hypothesis was rejected. Several mechanisms of the discoloration induced by MTA have been proposed. The first was that the gray color of the material itself is responsible for the discoloration. ${ }^{[15]}$ To address this concern, white MTA was introduced into the endodontic market; however, several reports indicated that even white MTA induces tooth discoloration. ${ }^{[12,15,16]}$ It was also proposed that metal oxides $(\mathrm{Fe}, \mathrm{Mn})$ could be responsible for the discoloration. ${ }^{[17]}$ Another suggestion is that the discoloration is due to chemical interaction of bismuth oxide $\left(\mathrm{Bi}_{2} \mathrm{O}_{3}\right)$ with dentin. ${ }^{[18,19]} \mathrm{Bi}_{2} \mathrm{O}_{3}$ is added to MTA to provide radiopacity. ${ }^{[20]}$ The discoloration induced by MTA is attributed to its progressive mass darkening due the presence of reduced black crystals of bismuth atoms. ${ }^{[21,22]}$ Among possible alternatives to $\mathrm{Bi}_{2} \mathrm{O}_{3}$, zirconium oxide $\left(\mathrm{ZrO}_{2}\right)$ was investigated as a candidate because of its adequate radiopacity and cost-effectiveness. Recent studies showed that a $\mathrm{ZrO}_{2}$-containing MTA induced less discoloration than MTAs containing $\mathrm{Bi}_{2} \mathrm{O}_{3} \cdot{ }^{[13,23]}$ According to the manufacturer, Endoseal contains both $\mathrm{Bi}_{2} \mathrm{O}_{3}$ and $\mathrm{ZrO}_{2}$ as radiopacifiers. It can be postulated that although Endoseal still has $\mathrm{Bi}_{2} \mathrm{O}_{3}$ as a constituent a considerable amount of $\mathrm{Bi}_{2} \mathrm{O}_{3}$ has been substituted by $\mathrm{ZrO}_{2}$ and as a result Endoseal showed little tooth discoloration in our study, comparable to that of AHplus. AHplus, a resin-based sealer, showed less discoloration than ProRoot, as expected $(P<0.05)$. In fact, AH26, an early version of AHplus, is well known to induce tooth darkening and is not recommended when aesthetics are important. ${ }^{[24,25]}$ This can be explained by the fact that $\mathrm{AH} 26$ contains $\mathrm{Bi}_{2} \mathrm{O}_{3}$ as a filler and radiopacifier whereas AHplus contains $\mathrm{ZrO}_{2}$. Taken together, these findings indicate that $\mathrm{Bi}_{2} \mathrm{O}_{3}$ can be considered a major cause of tooth discoloration, and it is best to avoid adding this radiopacifier to root canal sealers.

In the analysis of $L^{*}, a^{*}, b^{*}$ data, it was evident that $a$ and $b$ values were not affected by the application of root canal sealer. This may mean that the discoloration induced by sealers is not relevant to red/green color tendency or to yellow/blue tendency; rather, the discoloration induced by sealers seems to only 
influence the lightness of the tooth. Since $a, b$ values remained relatively stable over all time points, $\Delta L$ values are directly proportional to $\Delta E$ values.

In this study, we used bovine incisors to evaluate discoloration because of their many advantages over human teeth. First, we could easily obtain a sufficient number of intact bovine incisors. Second, the number of dentinal tubules per $\mathrm{mm}^{2}$ and diameter of tubules in coronal dentin of bovine incisors are similar to those of human teeth. ${ }^{[26]}$ Moreover, bovine incisors are wide enough to easily obtain standardized tooth samples. Although bovine incisors are widely used as specimens for in vitro studies, there are still limitations to their use and further investigations using human incisors are recommended.

\section{CONCLUSIONS}

Within the limitations of the present study, we conclude that a novel MTA-based root canal sealer, Endoseal, showed discoloration that is comparable to that of AHplus, and significantly lower than that with ProRoot. Although Endoseal appears to have little effect on tooth discoloration, further studies should be conducted to confirm its long-term color stability.

\section{Financial support and sponsorship}

This study was supported by Public institutions linked regional industry promotion project (Project: Technology Consulting for Medical Device Health Insurance Reimbursement Registration and Commercialization Support) of MOTIE (Korean Ministry of Trade, Industry and Energy).

\section{Conflicts of interest}

There are no conflicts of interest.

\section{REFERENCES}

1. Kim RJ, Shin JH. Cytotoxicity of a novel mineral trioxide aggregate-based root canal sealer [corrected]. Dent Mater J 2014;33:313-8.

2. Ahmed HM, Abbott PV. Discolouration potential of endodontic procedures and materials: A review. Int Endod J 2012;45:883-97.

3. Torabinejad M, Hong CU, Lee SJ, Monsef M, Pitt Ford TR. Investigation of mineral trioxide aggregate for root-end filling in dogs. J Endod 1995;21:603-8.

4. Torabinejad M, Hong CU, Pitt Ford TR, Kettering JD. Cytotoxicity of four root end filling materials. J Endod 1995;21:489-92.

5. Enkel B, Dupas C, Armengol V, Akpe Adou J, Bosco J, Daculsi G, et al. Bioactive materials in endodontics. Expert Rev Med Devices 2008;5:475-94.

6. Torabinejad M, Rastegar AF, Kettering JD, Pitt Ford TR. Bacterial leakage of mineral trioxide aggregate as a root-end filling material. J Endod 1995;21:109-12.

7. Lim ES, Park YB, Kwon YS, Shon WJ, Lee KW, Min KS. Physical properties and biocompatibility of an injectable calcium-silicate-based root canal sealer: In vitro and in vivo study. BMC Oral Health 2015;15:129.

8. Krastl G, Allgayer N, Lenherr P, Filippi A, Taneja P, Weiger R. Tooth discoloration induced by endodontic materials: A literature review. Dent Traumatol 2013;29:2-7.

9. Parirokh M, Torabinejad M. Mineral trioxide aggregate: A comprehensive literature review - Part I: Chemical, physical, and antibacterial properties. J Endod 2010;36:16-27.

10. Parirokh M, Torabinejad M. Mineral trioxide aggregate: A comprehensive literature review - Part III: Clinical applications, drawbacks, and mechanism of action. J Endod 2010;36:400-13.

11. Naik S, Hegde AH. Mineral trioxide aggregate as a pulpotomy agent in primary molars: An in vivo study. J Indian Soc Pedod Prev Dent 2005;23:13-6.

12. Belobrov I, Parashos P. Treatment of tooth discoloration after the use of white mineral trioxide aggregate. J Endod 2011;37:1017-20.

13. Yun DA, Park SJ, Lee SR, Min KS. Tooth discoloration induced by calcium-silicate-based pulp-capping materials. Eur J Dent 2015;9:165-70.

14. Joiner A. Tooth colour: A review of the literature. J Dent 2004;32 Suppl 1:3-12.

15. Felman D, Parashos P. Coronal tooth discoloration and white mineral trioxide aggregate. J Endod 2013;39:484-7.

16. Kohli MR, Yamaguchi M, Setzer FC, Karabucak B. Spectrophotometric analysis of coronal tooth discoloration induced by various bioceramic cements and other endodontic materials. J Endod 2015;41:1862-6.

17. Ioannidis K, Mistakidis I, Beltes P, Karagiannis V. Spectrophotometric analysis of coronal discolouration induced by grey and white MTA. Int Endod J 2013;46:137-44.

18. Marciano MA, Costa RM, Camilleri J, Mondelli RF, Guimarães BM, Duarte MA. Assessment of color stability of white mineral trioxide aggregate angelus and bismuth oxide in contact with tooth structure. J Endod 2014;40:1235-40.

19. Berger T, Baratz AZ, Gutmann JL. In vitro investigations into the etiology of mineral trioxide tooth staining. J Conserv Dent 2014;17:526-30.

20. Asgary S, Parirokh M, Eghbal MJ, Brink F. Chemical differences between white and gray mineral trioxide aggregate. J Endod 2005;31:101-3.

21. Vallés M, Mercadé M, Duran-Sindreu F, Bourdelande JL, Roig M. Color stability of white mineral trioxide aggregate. Clin Oral Investig 2013;17:1155-9.

22. Ioannidis K, Mistakidis I, Beltes P, Karagiannis V. Spectrophotometric analysis of crown discoloration induced by MTA- and ZnOE-based sealers. J Appl Oral Sci 2013;21:138-44.

23. Kang SH, Shin YS, Lee HS, Kim SO, Shin Y, Jung IY, et al. Color changes of teeth after treatment with various mineral trioxide aggregate-based materials: An ex vivo study. J Endod 2015;41:737-41.

24. Thomson AD, Athanassiadis B, Kahler B, Walsh L. Tooth discolouration: Staining effects of various sealers and medicaments. Aust Endod J 2012;38:2-9.

25. Zare Jahromi M, Navabi AA, Ekhtiari M. Comparing coronal discoloration between AH26 and ZOE sealers. Iran Endod J 2011;6:146-9.

26. Schilke R, Lisson JA, Bauss O, Geurtsen W. Comparison of the number and diameter of dentinal tubules in human and bovine dentine by scanning electron microscopic investigation. Arch Oral Biol 2000;45:355-61. 